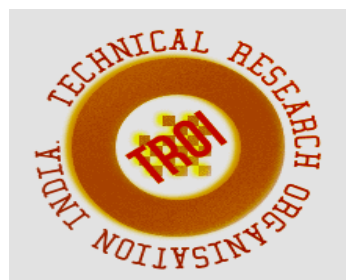

\title{
AN EMG BASED HAND GESTURE RECOGNITION SYSTEM USING SVM
}

\author{
Mrs. Vijayalakshmi G V ${ }^{1}$, Ajay J2 $\mathrm{J}^{2}$ Pavithra $\mathrm{S}^{3}$, Pooja Eronisha $\mathrm{A}^{4}$, Vanijayam $\mathrm{K}^{5}$ \\ ${ }^{1}$ Associate Professor, ECE dept., Dr. T. Thimmaiah Institute of Technology, K.G.F \\ ${ }^{2,3,4,5}$ Students, ECE dept., Dr. T. Thimmaiah Institute of Technology,K.G.F \\ 4poojaeronisha101919@gmail.com
}

\begin{abstract}
Hand gestures is one of the most powerful, immediate and natural means of communication. Electromyography (EMG) is the measure of electrical activity produced by the muscles which is usually represented as a function of time. EMG can be used in various applications including identifying neuromuscular diseases, sign language recognition, virtual reality, gesture to speech, robotics, and television control. The EMG based hand gesture recognition can help to develop good computer interface that increases the quality of life of the disable or aged people. The main purpose of this work is to identify the hand gestures that is classified and predefined using support vector machine (SVM). The raw EMG data is preprocessed and the statistical features are extracted, the extracted features are given to the support vector machine for training and classification and performance are evaluated using confusion matrix to determine the average classification accuracy as high as possible.
\end{abstract}

Keywords: hand gestures, EMG, support vector machine.

\section{INTRODUCTION}

Hand gestures are an integral part of human-human communication and can be leveraged for more natural human- machine interaction (HMI). They are a fast and effective physical medium for communicating with and controlling intelligent devices. Surface EMG is a non-invasive method of acquiring these signals by placing recording electrodes directly on the surface of the skin. The EMG signal is a biomedical signal that measures electrical currents generated in muscles during its contraction representing neuromuscular activities. The nervous system always controls the muscle activity (contraction/relaxation). Hence, the EMG signal is a complicated signal, which is controlled by the nervous system and is dependent on the anatomical and physiological properties of muscles.

\section{RELATED WORKS}

EMG-based gesture classification is a combination of flexible high density electrode array, and a dedicated biopotential acquisition device. Rachel Martina Edith and Bhargavi Haripriya [5] has demonstrated the study of surface EMG signal which is operating a power point. But, the signal which appears like properly acquired SEMG can be obtained by placing two electrodes on two different muscle. In the year 2016, Wahyu Caesarendra [6] made a case study in which Classification accuracy is an important issue in EMG classification for hand gesture identification. This study has shown an improvement of hand common gestures classification based on PCA and ANFIS. Which made the classification accuracy increased to $89.47 \%$. Tacar [7] proposed a work of surface EMG signals to control robotic hand movements which was also useful in the rehabilitation field. 
III.METHODOLOGY

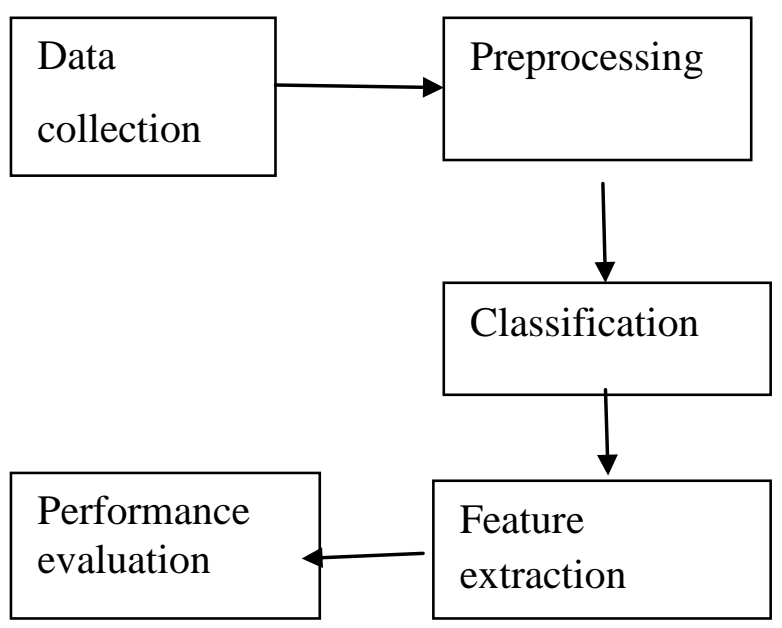

The goal here is to employ effective knowledge discovery and pattern recognition methods to increase the classification accuracy where ten classes of individual and combined finger movement are to be recognized. We have collected the EMG dataset from the EMG repository where, the dataset consists of ten classes of ten individual and combined figure movements. Then we performed a preprocessing task on the raw EMG data to enhance the performance of the EMG signal by removing artifacts like random noise, In preprocessing we performed filtering and segmentatiom of the EMG data, for filtering we used $4^{\text {th }}$ order butterworth bandpass filter which is obtained by cascading a highpass and a lowpass filter. The segmentation of EMG signal is necessary to understand the mechanisms related to muscle and nerve control. The data is divided into 40 equal parts of 500 samples each of $125 \mathrm{~ms}$ windowing size to make feature extraction process easy. Due to the stochastic nature of the EMG, any instantaneous sample of the EMG contains relatively little information about the overall muscle activity, hence some form of features must be extracted from the data. All of the extracted features from the two EMG channels were concatenated to form one large feature set using parallel feature fusion The final step in the EMG system employs a suitable classifier to recognize the signals from different classes of the fingers movements. We used Support Vector Machine (SVM) has a classifier to improve the performance in classification

\section{a) Data Collection}

IV IMPLEMENTATION

We collected the EMG dataset from a EMG repository which consists data of Eight subjects, six males and two females. The subjects were all normally limbed with no neurological or muscular disorders. Subjects were seated on an arm-chair, with their arm supported and fixed at one position to avoid the effect of different limb positions on the generated EMG signals.The EMG data was collected using two EMG channels (Delsys DE 2.x series EMG sensors) and processed by the Bagnoli Desktop EMG Systems from Delsys Inc. Ten classes of individual and combined fingers movements were implemented including: the flexion of each of the individual fingers, i.e., Thumb (T), Index (I), Middle (M), Ring (R), Little (L) and the pinching of combined Thumb-Index (T-I), Thumb-Middle ( $\mathrm{T}-\mathrm{M})$, Thumb-Ring (T-R), Thumb-Little ( $\mathrm{T}-$ $\mathrm{L})$, and finally the hand close (HC)..

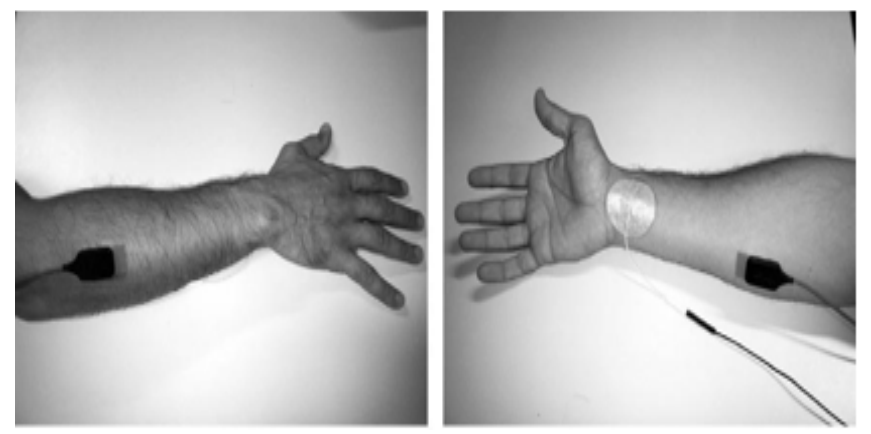

Fig. 1. Electrodes placement on the right forearm.

\section{b) Filtering}

The surface electromyographic (sEMG) signal contains the signal that originates in the muscle and various noise components which are endemic and unavoidable. These noise components contaminate the sEMG signal and may lead to an erroneous interpretation of the signal.

The useful data of EMG signal lies between the frequency of $20 \mathrm{~Hz}$ to $450 \mathrm{~Hz}$, where the other portions of the signal are considered to be unnecessary or the signal with less information. Hence, the signal should is bandpass filtered, we used butterworth bandpass filter of order 4 for filtering. The $4^{\text {th }}$ order butterworth bandpass filter is obtained by cascading two $2^{\text {nd }}$ order butterworth bandpass filter. Hence, the signals are filtered between 
$20 \mathrm{~Hz}$ to $450 \mathrm{~Hz}$ using a highpass and lowpass filter. A typical filtered data signal of single trial of a normally limbed male with hand close (HC) gesture which undergone filtering process is shown in Fig 2.

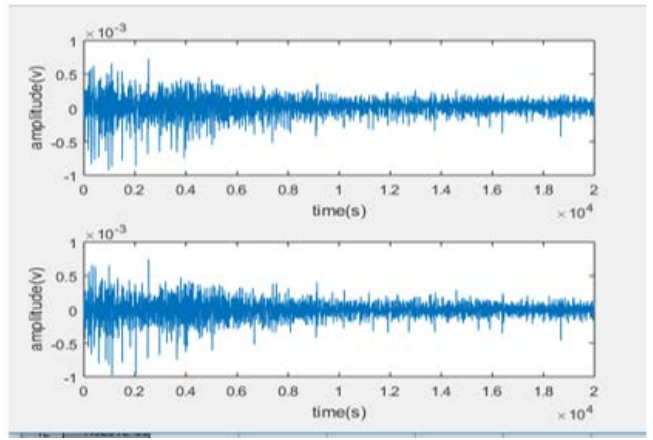

Fig 2: Filtered data

\section{c) Segmentation}

The segmentation of the signal is referred to decomposing a given signal into a simpler signals. The interference of the motor unit action potentials forms the EMG signal. Thus in order to understand the mechanism and functioning of the muscles. We have segmented the EMG signal. And the segmenting flow is after filtering total number of samples[ie, 20,000]from a single channel of a person for a single trail is divided by sampling rate of 4000 and it is scaled down to $5 \mathrm{sec}$, so it is clear that each trail is performed for 5sec. and also it is known that $5 \mathrm{sec}$ is equal to $5000 \mathrm{~ms}$, therefore again the 20,000 sample is divided by $5,000 \mathrm{~ms}$ and it is scaled down to 4 , and now this value is multiplied to $125 \mathrm{~ms}$ so the result obtained is 500 samples and hence the data is divided into 40 equal parts of 500 samples each of $125 \mathrm{~ms}$ windowing size to make feature extraction process easy[3]. The Fig 3 shows the segmented data of samples of 500 each from 1 to 2,000 samples of a normally limbed male of hand close (HC) gesture.

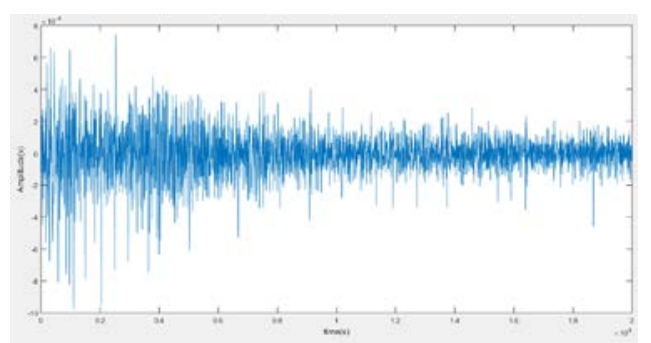

Fig 3: Segmented data

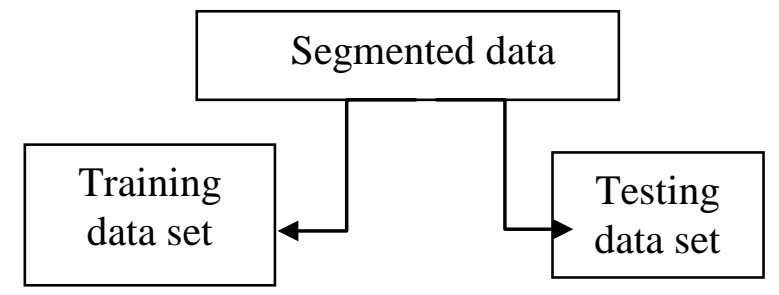

We have subdivided the segmented data into training and testing data set because SVM classifier is trained by training data set and testing data set is given as new input data to SVM classifier so that it classifies the gesture on basis of its training. Therefore from the segmented data 4 trail of each gesture of a single person is labeled as training data set and similarly 2 trail of each gesture is labeled as testing data set.

\section{d) Feature Extraction}

Feature extraction is a general term for methods of constructing combinations of the variables to get around these problems while still describing the data with sufficient accuracy. The purpose of feature extraction is not only to reduce the dimensionality but also to extract more useful/dominant information hidden in the signals by avoiding unnecessary or redundant info.

We have extracted the following features from the preprocessed data:

\begin{tabular}{|l|c|}
\hline Sl no & Feature Names \\
\hline 01 & Mean \\
\hline 02 & Median \\
\hline 03 & Standard deviation \\
\hline 04 & Covariance \\
\hline 05 & Mobility \\
\hline 06 & Complexity \\
\hline 07 & Zero crossing \\
\hline 08 & \\
\hline
\end{tabular}




\section{d) Feature Fusion}

It is known that the data is extracted from two homogenous sensors (Delsys DE 2.x series EMG sensors), hence fusion of the features can be done. Therefore we have fused the features of first segment of data from sensor 1 with the features of first segment of data from sensor 2. Similarly, the fusion is carried until the last segment.

For feature fusion we have tested by both serial feature fusion method and parallel feature fusion method, parallel fusion outcome was better than serial fusion outcome because of homogeneous property of sensor and column wise fusion. Hence we used parallel feature fusion technique.

\section{e) Classification}

A classifier utilizes values for independent variables (features) as input to predict the corresponding class to which an independent variable belongs (Pereira et al., 2009). A classifier has a number of parameters that require training from a training dataset. A trained classifier will model the association between classes and corresponding features and is capable of identifying new instances in an unseen testing dataset. An Support Vector Machine (SVM) is employed for the following classification method to demonstrate the effectiveness of the study proposed by classification technique. Training data points of any class as show in the below Fig.6.

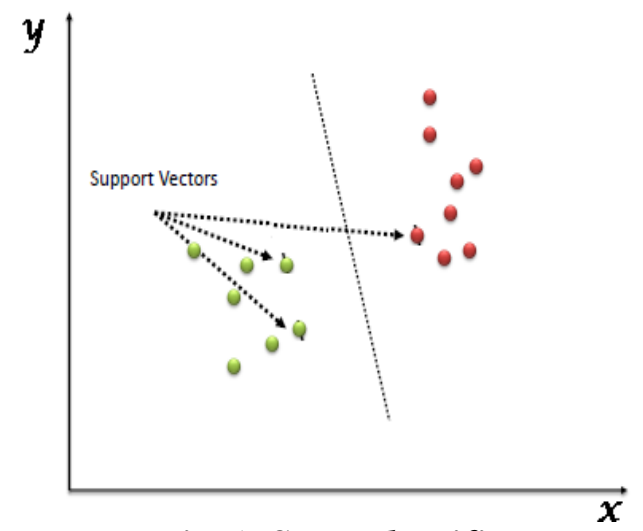

Fig.6: SVM classifier

A classification task usually involves separating data into training and testing sets. Each instance in the training set contains one target set i.e. the class labels. For the labeling of the target set, we have used binary values because SVM is a binary classifier the values will be either numeric or logic vectors each element of target specifies the group the corresponding row of training belongs to, training and target must have same number of rows. The input of the SVM classifier is the fused training features of a single gesture (i.e $40 \times 320$ ) and target set (i.e $40 \times 10$ ) which is given as input for training the SVM classifier.

\section{f) Performance evaluation}

The classified data with appropriate label should be tested and the accuracy is to be obtained. Hence, from the literature survey [2], we concluded that performance evaluation for increasing the accuracy using confusion matrix holds good and gives maximum accuracy. A confusion matrix is a table that is often used to describe the performance of a classification model (or "classifier") on a set of test data for which the true values are known. The confusion matrix itself is relatively simple to understand, but the related terminology can be confusing. Confusion matrix contains information about actual and predicted classifications done by a classification system. Performance of such systems is commonly evaluated using the data in the matrix.

\section{EXPERIMENTS AND RESULTS}

The data which had been collected from the authenticated source is filtered using Butterworth fourth order bandpass filter to remove the noise and it is segmented. From the segmented data, stastical features are extracted for each subject, the SVM classifier was trained on the extracted features from the first four trials for all movements and then tested with the data extracted from the remaining two trails for all movements, kernel type: radial basis function.

The diagonals of the confusion matrices were averaged in order to investigate the different class's recognition capability of the proposed system across the 3 subjects.

In the current system with just 2 EMG channels, the classifier performed nearly in a similar manner achieving an average class wise accuracy of $90.83 \%$ which seems acceptable in comparison to other work from the literature.

\section{CONCLUSION}

A two channel EMG pattern recognition system was proposed in this paper to classify individual and combined finger movements. 
Various features were extracted from the two channels and reduced in dimensionality using parallel method. In order to obtain enhanced output classification decisions made by the current EMG pattern recognition system, SVM classifier of kernel radial basis function (rbf) is proposed. This research proved the feasibility of the proposed system achieving 90.83\% classification accuracy results with the SVM classifier. The current results suggest the success of the two EMG channels system in classifying three different individual and combined finger movements.

\section{REFERENCES}

[1] Hjorth, B. (1970). EEG analysis based on time domain parameters. Electroencephalography and Clinical Neurophysiology, 29(3), 306-310.

[2] Englehart,K.(1998).Signal representation for classification ofthetransient myoelectric ignal. Ph.D. Dissertation. New Brunswick, Canada: Department ofElectrical and Computer Engineering, University of New Brunswick.

[3]_Jan Sedlak, Daniel Suplak, 2013 International Conference on Applied Electronics.
[4] Rami N. Khushaba, SarathKodagoda, MaenTakruri, GaminiDissanayake "Toward improved control of prosthetic fingers using surface electromyogram (EMG) signals”.

[5] Rachel Martina Edith and BhargaviHaripriya(2015) presented a work on, "Gesture Recognition Using Real Time EMG”SRM University. IEEE Sponsored $2^{\text {nd }}$ International Conference on Inovation in Information, Embedded and Communication.

[6] WahyuCaesarendra,

TeroehTjahjowidodo and Daniel Pamugkas(2017), "EMG based Classification of Hand Gestures using PCA and ANFIS”, International Conference of Innovation of Embedded Application.

[7] Tacar and A Jaimes(2015) "Real time tracking algorithm for human hand motion. powered hand prosthetics is a field which uses EMG signals for design" IEEE Sponsored $2^{\text {nd }}$ International conference on Innovation and Application Based Security System. 